\title{
Chapter 9. Hypertension in women
}

\author{
Hypertension Research (2009) 32, 63-65; doi:10.1038/hr.2008.7
}

\section{POINT 9}

Pregnancy-induced hypertension

1. Hypertension observed in pregnancy should be understood as hypertension under special conditions.

2. Hypertension is divided into two types: one which occurs after the 20th week of gestation and the other which exists before pregnancy.

3. In pregnancy-induced hypertension:

i. Hypertension ( $\geqslant 140 / 90 \mathrm{~mm} \mathrm{Hg}$ ) with or without proteinuria occurring after the 20th week of gestation but resolving by the 12 th week of postpartum is defined as pregnancy-induced hypertension.

ii. Mild hypertension in pregnancy should not be treated aggressively.

iii. Severe hypertension in pregnancy should be treated.

iv. See Table 9-2 for definitions of mild and severe hypertension.

4. In pregnant women with pre-existing hypertension, any changes to antihypertensive drug treatment should be made with caution.

5. Methyldopa, hydralazine hydrochloride and labetalol are primary antihypertensive drugs, but $\mathrm{Ca}$ channel blockers may also be used cautiously with sufficient informed consent of the patient.

6. Pregnancy-induced hypertension is a contraindication to the use of ACE inhibitors and angiotensin II receptor blockers (ARB).

\section{Postmenopausal hypertension}

7. As blood pressure may be increased by oral contraceptives or hormone replacement therapy, close observation is needed.

8. The pathophysiology and treatment of hypertension in women, including postmenopausal women, may differ from those in men.

The tendency to emphasize gender differences is growing in medical fields. ${ }^{564}$ With regard to cardiovascular disease, in particular, vast differences in the pathogenic processes and their outcomes have been clarified. ${ }^{565}$ Recent epidemiological studies have shown that pregnancy and childbirth are important as basic differences and that they exert marked effects on cardiovascular disease in women. ${ }^{566}$ However, the prevalence of hypertension increases rapidly in women after meno- pause and becomes comparable to that in men after about 65 years of age (Figure 9-1).

In this chapter, two major categories of hypertension in women, that is, hypertension related to pregnancy and hypertension in the postmenopausal period, are discussed.

\section{1) HYPERTENSION RELATED TO PREGNANCY}

Hypertension observed during pregnancy is defined as pregnancyinduced hypertension. Table 9-1 shows the typing of the disease and Table 9-2 shows the classification of hypertension. Hypertension in pregnant women is also classified according to symptoms and the time of onset, but, for this, readers should refer to the definition and classification of pregnancy-induced hypertension by the Japan Society for the Study of Hypertension in Pregnancy. ${ }^{567}$ Hypertension in pregnant women may be pregnancy-induced, essential or secondary, and careful examination is necessary. As with other types of hypertension, 24-h ambulatory blood pressure monitoring has been reported to be useful for predicting proteinuria, neonate weight and the possibility of premature birth. ${ }^{568}$

a. Antihypertensive drug treatment for hypertension in pregnant women

Mild pregnancy-induced hypertension. There are various difficulties in designing and securing subjects for randomized controlled studies for evaluating the appropriateness of antihypertensive drug treatment in patients with mild pregnancy-induced hypertension. Therefore, related evidence is scarce both in Japan and overseas. However, meta-analysis to date has shown that the transition rate of mild hypertension (systolic blood pressure 140-159 $\mathrm{mm} \mathrm{Hg}$, diastolic pressure $90-109 \mathrm{~mm} \mathrm{Hg}$ ) with no target organ damage to severe hypertension (systolic blood pressure $\geqslant 170 \mathrm{~mm} \mathrm{Hg}$, diastolic pressure $\geqslant 110 \mathrm{~mm} \mathrm{Hg}$ ) was $50 \%$ or less in the group that received antihypertensive drug treatment compared with the control group, but there was no difference in the frequency of its progression to pre-eclampsia, and there was no significant difference in the frequency of perinatal death or premature delivery. ${ }^{569}$ On the basis of these results, antihypertensive treatment for mild hypertension during pregnancy is often viewed negatively. ${ }^{570} \mathrm{In}$ mild hypertension, blood pressure often decreases after the discontinuation of antihypertensive medication that had been taken before pregnancy, but the re-introduction of antihypertensive treatment is recommended at a systolic blood pressure of about $160 \mathrm{~mm} \mathrm{Hg}$ and a diastolic blood pressure of about $110 \mathrm{~mm} \mathrm{Hg}$. ${ }^{571}$

Moreover, according to meta-analyses regarding the effects on fetuses, the results of studies using any antihypertensive drug indicated a direct relationship between a decrease in mean blood pressure in the range of $107-129 \mathrm{~mm} \mathrm{Hg}$ and low birth weight. ${ }^{572}$ 

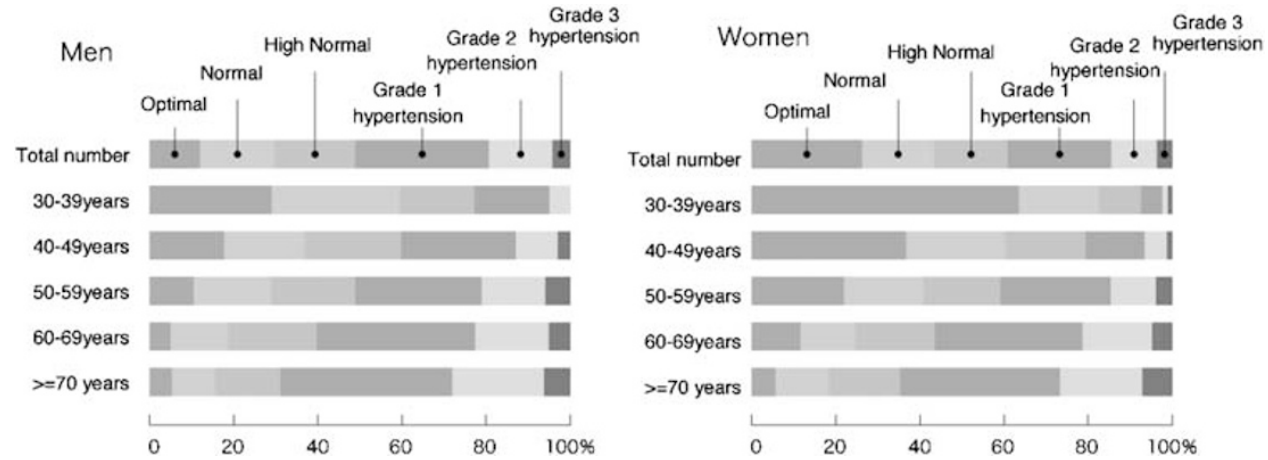

Figure 9-1 Percentage of various blood pressure categories by gender and age.

\section{Table 9-1 Definition and classification of pregnancy-induced hypertension}

Definition

(i) Gestational hypertension

Hypertension (systolic blood pressure $\geqslant 140 \mathrm{~mm} \mathrm{Hg}$ or diastolic pressure $\geqslant 90 \mathrm{~mm} \mathrm{Hg}$ ) with or without proteinuria occurring after the 20th week of gestation but resolving by the 12th week of postpartum is defined as pregnancy-induced hypertension. It is essential for diagnosis to exclude any background disease that could cause hypertension or proteinuria.

(ii) Pre-eclampsia

Hypertension (systolic blood pressure $\geqslant 140 \mathrm{~mm} \mathrm{Hg}$ or diastolic blood pressure $\geqslant 90 \mathrm{~mm} \mathrm{Hg}$ ) with proteinuria (basically $\geqslant 300 \mathrm{mg}$ per day) occurring after the 20th week of gestation but resolving by the 12 th week of postpartum.

(iii) Eclampsia

Seizure occurring after the 20th week of gestation. Exclusion of epilepsy as well as other neurogenic disorders is essential.

(iv) Superimposed pre-eclampsia

(a) Chronic hypertension diagnosed before pregnancy or before the 20th week of gestation, along with proteinuria emerging after the 20th week of gestation.

(b) Aggravation of pre-existing (before pregnancy or before the 20th week of gestation) hypertension and proteinuria after the 20th week of gestation. Either one or both aggravations are acceptable.

(c) Hypertension emerging after the 20th week of gestation in patients with pre-existing renal diseases that manifest solely proteinuria.

\section{Table 9-2 Subclass by symptoms}

\section{Severity}

Mild pregnancy-induced hypertension

Systolic blood pressure $>140 \mathrm{~mm} \mathrm{Hg}$ but not exceeding $160 \mathrm{~mm} \mathrm{Hg}$ and/or diastolic blood pressure $>90 \mathrm{~mm} \mathrm{Hg}$ but not exceeding $110 \mathrm{~mm} \mathrm{Hg}$.

Proteinuria $>300 \mathrm{mg}$ per day but not exceeding $2 \mathrm{~g}$ per day.

Severe pregnancy-induced hypertension

Systolic blood pressure $>160 \mathrm{~mm} \mathrm{Hg}$ and/or diastolic blood pressure

$>110 \mathrm{~mm} \mathrm{Hg}$.

Proteinuria $>2$ g per day.

Correlation of 24-h urine quantitation and randomly sampled urine assay is substantially poor. Thus, a 24-h urine sample should be used. Under the

circumstances that only randomly sampled urine is available, repeated test results of $3+$ are considered to be severe.

Severe pregnancy-induced hypertension. Severe pregnancy-induced hypertension is often treated on the basis of experience without sufficient evidence. The presence of target organ damage is a positive indication for antihypertensive treatment, ${ }^{573}$ and as high blood pressure can cause damage to the mother's brain, cardiovascular system, kidneys, and so on, antihypertensive treatment must be initiated promptly. However, the primary objective of treatment for severe hypertension is the prevention of damage to the mother's organs, and there is insufficient data on whether the treatment is also beneficial to fetuses. ${ }^{574}$

On the basis of these opinions, the ESH/ESC Guidelines recommend that antihypertensive drug treatment should be started when systolic blood pressure is $\geqslant 150 \mathrm{~mm} \mathrm{Hg}$ and diastolic blood pressure is $\geqslant 95 \mathrm{~mm} \mathrm{Hg}$, and that it should be administered more aggressively (systolic blood pressure $\leqslant 140 \mathrm{~mm} \mathrm{Hg}$, diastolic blood pressure $\leqslant 90 \mathrm{~mm} \mathrm{Hg}$ ) if there is a history of pregnancy-induced hypertension or hypertension before pregnancy. ${ }^{66}$

Pregnancy with pre-existing hypertension. Controlling blood pressure at $\leqslant 140 / 90 \mathrm{~mm} \mathrm{Hg}$ allows a normal pregnancy and delivery. If the patient is undergoing multiple-drug antihypertensive treatment, has target organ damage, has had delivery at a mature age ( $\geqslant 35$ years), or is obese or diabetic, prompt consultation with a hypertension specialist and an obstetrician is necessary.

Pre-eclampsia. Pre-eclampsia should be understood as a multiple organ disease. It causes HELLP syndrome (hemolysis, elevated liver enzymes and low platelets syndrome), pulmonary edema, renal failure, disseminated intravascular coagulation, cerebrovascular disease, abruptio placentae, and so on, as well as eclampsia in the mother. It also increases the mortality and morbidity rates of infants through placental dysfunction.

\section{b. Antihypertensive drugs}

The drugs that were used most frequently were methyldopa and hydralazine hydrochloride. Since the safety of these drugs has been sufficiently established, they have been used as primary antihypertensive drugs for the treatment of hypertension in pregnancy to date. ${ }^{575}$

However, as these drugs will not be in routine use until their use is supported by Western guidelines, the usefulness of Ca channel blockers is only gradually being recognized. ${ }^{66}$ In Japan, pregnancy is a contraindication to the use of many Ca channel blockers, but as there have been few reports of serious adverse effects ${ }^{576}$ and as they are recommended by overseas guidelines, their use with sufficient informed consent may be permitted in the future. ${ }^{564}$

Among $\beta$-blockers, labetalol hydrochloride, an $\alpha \beta$-blocker, is primarily used.

Pregnancy is considered to be a contraindication to the use of ACE inhibitors and angiotensin II receptor blockers (ARBs), ${ }^{577}$ as both 
have been reported to cause various disorders in fetuses, such as oligoamnios, renal insufficiency and growth disorders. ${ }^{578}$ However, according to the results from women who unexpectedly became pregnant during their use, these disorders did not always occur, and their frequency was relatively low. ${ }^{579}$ Nevertheless, ACE inhibitors and ARBs are still contraindicated for use in pregnant women.

Concerning diuretics, pre-eclampsia is theoretically accompanied by hemoconcentration and a decrease in the circulating blood volume, and diuretics are likely to exacerbate these conditions, causing a decrease in the placental blood flow. Therefore, diuretics must not be used, in principle, in patients with pre-eclampsia unless there is pulmonary edema or signs of heart failure. On the other hand, if diuretics have been used before pregnancy, their continued use has not been reported to cause a marked decrease in the placental blood flow. ${ }^{580}$

\section{c. Non-drug antihypertensive treatments}

None of the non-drug treatments, such as salt restriction, weight control and calcium supplementation, has been shown to be effective in the control of hypertension in pregnancy. A low dose of aspirin, however, has been suggested to prevent the progression of preeclampsia occurring within the 20-28th week of gestation. ${ }^{581}$

Magnesium sulfate $\left(\mathrm{MgSO}_{4}\right)$, which is not an antihypertensive drug, is widely used for the prevention of eclampsia during the induction of delivery in patients with impending eclampsia. ${ }^{582} \mathrm{An}$ intravenous injection of $\mathrm{MgSO}_{4}$ (4g of $\mathrm{MgSO}_{4}$ in $100 \mathrm{ml}$ of $5 \%$ glucose solution over $10-15 \mathrm{~min}$ ) followed by its administration at $1-2 \mathrm{~g} \mathrm{~h}^{-1}$ is considered to be the most effective treatment.

\section{d. Breast feeding}

Caution is needed when breast feeding because a small percentage of most antihypertensive drugs is secreted in breast milk. The discontinuation of antihypertensive medication should be considered if the diastolic blood pressure is $<100 \mathrm{~mm} \mathrm{Hg}$. However, if hypertension is severer, priority should be given to antihypertensive drug treatment, and breast feeding should be discontinued. Among antihypertensive drugs, captopril, an angiotensin converting enzyme inhibitor, and hydrochlorothiazide, a diuretic, are compatible antihypertensive drugs with breastfeeding. ${ }^{583}$

\section{HYPERTENSION RELATED TO POSTMENOPAUSAL WOMEN}

Various changes, including changes in the cardiovascular system, occur in postmenopausal women. ${ }^{584}$ Dyslipidemia, for example, markedly affects the cardiovascular system. ${ }^{585}$ Blood pressure is considered to increase after menopause by some, but is not considered to change markedly by others, and therefore no consensus has been reached. ${ }^{586}$ It seems certain, however, that a notable percentage of women who showed a normal or low blood pressure until about 35 years of age exhibit a rapid increase in blood pressure or are diagnosed as hypertensive after menopause. ${ }^{587}$ In addition, the possibility of white coat hypertension must not be forgotten in postmenopausal women. Furthermore, various changes (for example, weight gain and changes in hormone levels such as estrogen) occur in this period, possibly facilitating increases in blood pressure. ${ }^{587}$ Psychological factors may also increase the changeability of the blood pressure and make sufficient antihypertensive drug treatment difficult. Hormones, psychotropic drugs or Kampo drugs are used for the treatment of such cases of hypertension in the postmenopausal period, but the results are unsatisfactory. ${ }^{588}$ Estrogen is used for the treatment of postmenopausal syndrome but has been considered to cause adverse effects at a high dose, such as an increase in blood pressure and thromboembolism. ${ }^{589}$ Although increased angiotensinogen synthesis and the subsequent enhanced production of angiotensin II are speculated to contribute to the estrogen-induced elevation of blood pressure, the precise mechanism remains unclear. In postmenopausal women, hormone replacement therapy is not considered to affect the blood pressure, but it may cause an increase in blood pressure in patients with a predisposition to hypertension, so that a follow-up with blood pressure measurement every few months is necessary. ${ }^{590}$ According to the Women's Health Initiative (WHI) report, estrogen increased cardiovascular events in postmenopausal women; ${ }^{589}$ therefore its careful use at a low dose is now recommended and so estrogen-induced hypertension has become rare. ${ }^{591}$ In Japan, medroxyprogesterone acetate (progestogen), which is considered to have no marked effect on the blood pressure, is employed as a progesterone to be used concomitantly with estrogen for hormone replacement therapy.

A point to be noted here is that responses to renin-angiotensin system inhibitors differ between men and women. Blood pressure may be easier to control with renin-angiotensin system inhibitors in men than in women. ${ }^{592}$ Although the cause of this difference is unclear, there is a possibility that the balance between estrogen and progesterone is disturbed in postmenopausal women and that the resultant tendency of water retention causes a condition resembling saltdependent hypertension. ${ }^{593}$ This makes diuretics a promising candidate as the first choice, but deciding upon which drug is appropriate for the treatment of postmenopausal women or what target should be set for the control of their blood pressure is a subject for future evaluation. ${ }^{594}$ Moreover, obesity has recently been suggested to be an important risk factor for estrogen-dependent tumors, such as cancers of the uterine body and breast as well as cardiovascular and metabolic abnormalities, not only in men but also in postmenopausal women. ${ }^{595}$ In addition, a close relationship between a history of pregnancyinduced hypertension and hypertension or nephropathy in postmenopausal women is being clarified. ${ }^{596,597}$

\section{Citation Information}

We recommend that any citations to information in the Guidelines are presented in the following format:

The Japanese Society of Hypertension Guidelines for the Management of Hypertension (JSH 2009). Hypertens Res 2009; 32: 3-107.

Please refer to the title page for the full list of authors. 